\title{
La función social de las bibliotecas públicas:
}

\section{la perspectiva profesional}

\author{
Eduardo Romero-Sánchez \\ Universidad de Murcia. Facultad de Educación, Murcia, España \\ eromero@um.es \\ José-Antonio Gómez-Hernández \\ Universidad de Murcia. Facultad de Comunicación y Documentación, Murcia, España \\ jgomez@um.es \\ Manuel Hernández-Pedreño \\ Universidad de Murcia. Facultad de Economía y Empresa. Departamento de Sociología, Murcia, \\ España \\ manuel@um.es
}

DOI: https://doi.org/10.26512/rici.v12.n2.2019.20642

Recebido/Recibido/Received: $2018-12-10$

Aceitado/Aceptado/Accepted: 2018-12-20

Resumen: El objetivo de esta investigación es analizar el potencial y las limitaciones que presentan las bibliotecas públicas para el desarrollo de la función social. La metodología se ha basado en la aplicación de dos técnicas cualitativas: la entrevista y el grupo de discusión. Las entrevistas se realizaron a diez expertos, seleccionados por su alta experiencia profesional en entornos bibliotecarios mediante un cuestionario virtual estructurado en 9 cuestiones vinculadas con la función social y el rol a desempeñar por las bibliotecas públicas. La opinión de los bibliotecarios de la Región de Murcia se obtuvo mediante tres grupos de discusión constituidos por el tamaño de la población de sus municipios, en los que se analizó su asunción de la función, los recursos humanos y materiales, necesidades formativas y tipo de usuarios, entre otros relacionados con este servicio. Los discursos obtenidos confirman la existencia de diferentes concepciones de la función social de la biblioteca, materializados en el distinto compromiso y estando mediados por aspectos vinculados con los recursos, el tipo de usuarios o la formación del profesional bibliotecario; sin olvidar, la relevancia de las actitudes, tanto del personal bibliotecario como de los responsables políticos. Sin duda, estos condicionantes pueden minimizarse desarrollando diversas estrategias de liderazgo y colaboración con otros agentes, externos e internos.

Palabras clave: biblioteca social. biblioteca pública. Estudio de usuarios. función social. investigación cualitativa. usuarios vulnerables.

Social role and function of public libraries: the librarian point of view

Abstract: Public libraries already assume, as one of their priorities, to facilitate access to information and digital literacy, understood as a requirement for the basic human right to information. Because of it, libraries facilitate information and digital literacy services devoted to improving users' competences for a critical, reflexive and intentional use of information. This function has been recently valued as a compensatory factor of the digital divide of vulnerable user, and a way to reduce their degree of social exclusion. The aim of this research is to analyze the potential and limitations that public libraries present when design and apply information and digital literacy services for their users. The methodology is 
based on qualitative techniques as the interview and the discussion groups. Interviews to librarians were carried out to ten considered expert ones using a questionnaire including ten questions linked with the social function and about the role that public libraries should play for social inclusion of citizens. Points of view of public librarians of the Region of Murcia was obtained by means of three groups of discussion selected according with population size of cities where are located their libraries following the same script, structured according with topics about social inclusion services as their human and material resources, competences needed by librarians, typology of users, and so on. The discourses obtained confirm that librarians have different conceptions about the social function of the library and, therefore, manifest a different degree of commitment to this role. This commitment is conditioned by aspects such as resources, the type of users or the training of the librarian and, above all, the attitudes of librarians as well as local political leaders are very relevant. To minimize these restrictions, a set of leadership and collaboration strategies must be developed with other agents, external and internal, which are described in the document.

Keywords: public library. qualitative research. social function. social library. User studies. vulnerable users.

\section{A função social das bibliotecas públicas: a perspectiva profissional}

Resumo: O objetivo desta pesquisa é analisar o potencial e as limitações das bibliotecas públicas para o desenvolvimento da função social. A metodologia foi baseada na aplicação de duas técnicas qualitativas: a entrevista e o grupo de discussão. As entrevistas foram realizadas com dez especialistas, selecionados por sua alta experiência profissional em ambientes de biblioteca, por meio de um questionário virtual estruturado em nove questões relacionadas à função social e ao papel a ser desempenhado pelas bibliotecas públicas. A opinião dos bibliotecários da Região de Múrcia foi obtida por meio de três grupos de discussão constituídos pelo tamanho da população de seus municípios, que analisaram sua assunção da função, recursos humanos e materiais, necessidades de treinamento e tipo de usuários, entre outros relacionados a este serviço. Os discursos obtidos confirmam a existência de diferentes concepções da função social da biblioteca, materializadas nos diferentes compromissos e sendo mediadas por aspectos relacionados aos recursos, ao tipo de usuários ou à formação do bibliotecário; sem esquecer, a relevância das atitudes, tanto do pessoal bibliotecário como dos líderes políticos. Sem dúvida, essas condições podem ser minimizadas pelo desenvolvimento de várias estratégias de liderança e colaboração com outros agentes, externos e internos.

Palavras-chave: biblioteca social. biblioteca pública. Estudo de usuários. função social. pesquisa qualitativa. usuários vulneráveis.

\section{Introducción}

Toda biblioteca pública es por esencia "social", pues es una institución abierta a todos los miembros de la comunidad en que se inserta, financiada por administración pública, y que tiene como fin facilitar un derecho básico universal, el acceso a la información. En este trabajo queremos analizar cómo se materializa esta función social de la biblioteca, a partir de una conceptualización de en qué consiste, cómo la ejercen los profesionales de las bibliotecas públicas, qué actitudes y competencias consideran necesarias, qué necesidades y limitaciones perciben para su desarrollo y con qué condicionantes de carácter organizativo o político se pueden encontrar. Se tratará por tanto de articular cómo se relacionan el discurso teórico sobre lo que es ser una biblioteca social, y la visión de profesionales y técnicos en un contexto bibliotecario, económico y social concreto en que se lleva a cabo. 


\section{Enfoques teóricos y práctica de la función social en el contexto español}

El concepto de biblioteca social o la función social de la biblioteca puede abarcar varios posibles enfoques o dimensiones, que creemos que tienen un carácter complementario y que hemos articulado según la Tabla 1.

Tabla 1. Dimensiones de la función social de la biblioteca

\begin{tabular}{|l|l|}
\hline \multicolumn{2}{|c|}{ Dimensiones de la función social de la biblioteca } \\
\hline Extensa o general & $\begin{array}{l}\text { Algo para tod@s, abierta de forma universal y gratuita como } \\
\text { espacio público }\end{array}$ \\
\hline Compensatoria o inclusiva & $\begin{array}{l}\text { Atención a personas y colectivos en riesgo de exclusión } \\
\text { informacional, digital o social }\end{array}$ \\
\hline Participación y colaboración & $\begin{array}{l}\text { Fomentar la gestión participativa y los usos grupales, } \\
\text { comunitarios y colaborativos }\end{array}$ \\
\hline Sostenibilidad local y global & $\begin{array}{l}\text { Contribución al desarrollo socioeconómico y laboral local y } \\
\text { global de acuerdo con los ODS2030 }\end{array}$ \\
\hline
\end{tabular}

Fuente: Elaboración propia

Así, en primer lugar, ser social significa que la biblioteca es de todos y para todos. La biblioteca pública debe ofrecer algo a cualquier persona que se acerque a ella, sin exclusión por ningún motivo. Ello es consecuencia de su carácter público por su financiación, sus fines y la gratuidad de la prestación de sus servicios. Esta primera sería una concepción general o extensa de la Biblioteca Social, y de ella se derivaría el que se la ha considerado un espacio público, "un tercer lugar" que permite el encuentro de todas las personas más allá de los ámbitos familiar y laboral (ELMBORG, 2011).

En segundo lugar, en un mundo desigual con muchas personas en riesgo de exclusión, la función social se relaciona con los esfuerzos por ser un servicio compensatorio de la desigualdad de oportunidades informativas, educativas o culturales de determinados sectores vulnerables, como se refleja en el desarrollo de pautas para colectivos como el de las personas sin hogar (IFLA, 2017). La brecha o exclusión digital por falta de acceso y de competencias es un factor que incrementa el resto de componentes de la exclusión (GÓMEZ-HERNÁNDEZ, HERNÁNDEZ-PEDREÑO, ROMERO-SÁNCHEZ, 2017), y desde este punto de vista, esta concepción de la biblioteca social da predominio al carácter compensatorio y la acción proactiva con las personas y colectivos vulnerables: sería la biblioteca inclusiva.

En tercer lugar, al menos, el que la biblioteca sea social tiene que ver con un enfoque de su gestión y sus servicios: incluir la participación ciudadana, favorecer los espacios "sociales" destinados a encuentro de distintas personas, dar oportunidades de socialización para quienes están en situación de soledad (DOMíNGUEZ-SANJURJO, 2017), facilitar el trabajo colaborativo, la creación, la reunión de los grupos de la comunidad y la innovación social. En 
este caso la biblioteca es social en su forma de abrirse a las nuevas formas de trabajo, orientando los servicios y los espacios más a las personas que a las colecciones, materializando ello también en la configuración de sus infraestructuras e instalaciones, y asumiendo los principios del gobierno abierto en su organización. El eje de este enfoque sería la participación y la colaboración.

$Y$ en cuarto lugar -al menos- se podría englobar en la función social el compromiso con el desarrollo sostenible local y global, y ello explica que las bibliotecas públicas apoyen a la economía o la inserción laboral de su entorno próximo (CASTILLO-FERNÁNDEZ, 2010) y estén trabajando por los Objetivos de Desarrollo Sostenible de 2030. Y tanto en relación con la meta 16.10 relativa al derecho universal a la información que se afirmó en la Declaración de Lyon (IFLA, 2014), como por su aportación transversal a todos los objetivos (IFLA, 2016). Así, en su discurso inicial como presidenta de IFLA, Pérez-Salmerón (2017) manifestaba esta responsabilidad: "ser el engranaje de los motores para un real y visible cambio en el desarrollo y empoderamiento de nuestra sociedad". En este sentido, la biblioteca es social en tanto desea contribuir a la mejora y la sostenibilidad local y global, en un contexto de desequilibrios crecientes que cuestionan la viabilidad futura como sociedad: La sostenibilidad social y ambiental sería el rasgo clave en este enfoque.

Lógicamente estas dimensiones de la biblioteca que componen su carácter social no se dan aisladas, sino que están presentes y se entremezclan en distintos grados, pudiendo generar un conjunto de beneficios a los individuos en particular y a la comunidad en general, descritos en el Estudio sobre el valor de las bibliotecas públicas en la sociedad realizado en la Diputación de Barcelona (TOGORES, 2014) (Tabla 2).

Tabla 2. Beneficio social de las bibliotecas

\begin{tabular}{|l|l|l|}
\hline Eje & \multicolumn{1}{|c|}{ Perspectiva individual } & \multicolumn{1}{c|}{ Perspectiva comunitaria } \\
\hline Cultural & $\begin{array}{l}\text { - Fomento del hábito y de la } \\
\text { competencia lectora } \\
\text { - Acceso universal al conocimiento y } \\
\text { a la lectura }\end{array}$ & $\begin{array}{l}\text { - Preservación y difusión de la } \\
\text { memoria cultural local } \\
\text { Progreso cultural y artístico } \\
\text { local }\end{array}$ \\
\hline Social & $\begin{array}{l}\text { - Inclusión social } \\
\text { Inclusión de personas con } \\
\text { discapacidad } \\
\text { - Inclusión de la diversidad cultural }\end{array}$ & $\begin{array}{l}\text { - Cohesión social } \\
\text { Capital social } \\
\text { Revitalización del espacio } \\
\text { público }\end{array}$ \\
\hline Económico & $\bullet$ Fomento de la inclusión laboral & $\begin{array}{l}\text { Fomento del progreso } \\
\text { económico local }\end{array}$ \\
\hline Educativo / Informativo & $\begin{array}{l}\text { - Alfabetización informacional } \\
\text { Inclusión digital }\end{array}$ & $\bullet$ Sociedades mejor informadas \\
\hline
\end{tabular}

Fuente: Togores (2014). 
Ahora bien, la puesta en práctica se da siempre en contextos muy concretos, dando lugar -explícita o implícitamente- a concepciones muy distintas de cómo ejercer la función social y con qué prioridades, debiéndose considerar cuatro aspectos fundamentales:

- Las características sociodemográficas y económicas de los municipios.

- Los recursos humanos y materiales de las bibliotecas públicas, frecuentemente afectados de precariedad y escasez de personal (poco más de dos bibliotecarios a tiempo completo de media o municipios pequeños con un solo bibliotecario).

- La estructura y organización de los servicios sociales y educativos municipales y la intervención de agentes del tercer sector en la comunidad, que asuman total o parcialmente prestaciones y servicios a los que pueden también contribuir las bibliotecas

- Además, factores como la reciente crisis económica han influido en la orientación de las prioridades a atender, haciendo incrementar la función social "compensatoria" de las bibliotecas (MERLO-VEGA, CHU, 2015) quizás frente a la atención de sus otras dimensiones.

Si se atiende al Grupo de Trabajo Funcion Social de la Biblioteca (2017) del Ministerio de Cultura, sin dejar de lado el segundo enfoque descrito (compensatorio o inclusivo para contribuir a reducir la desigualdad y crear oportunidades) una biblioteca debe ser "social" en el sentido más amplio, que sumaría todas las dimensiones: a) un lugar de encuentro y atención a cualquier persona de la comunidad; b) que desarrolla servicios atendiendo a la diversidad de edades, necesidades e intereses; c) que incorpora la participación ciudadana; d) que fomenta la comunicación de su labor en la sociedad; y e) que colabora con otras entidades públicas y del tercer sector para el logro de sus fines, pues son compartidos por muchos de los agentes públicos y privados que están en su mismo entorno.

La Fundación Biblioteca Social ha procurado desde su origen identificar y difundir como ejemplos de buena práctica los servicios a grupos específicamente desfavorecidos (D’ALÒS-MONER, BAILAC, HERNÁNDEZ-SÁNCHEZ, 2015). A través de su Premio Anual se nos han dado a conocer varias decenas de proyectos de contribución de las bibliotecas a la inclusión de colectivos específicos: adolescencia y juventud, discapacidad, infancia, inmigración y refugiados, minorías étnicas, personas mayores, pobres y con enfermedad mental (FUNDACIÓN BIBLIOTECA SOCIAL, 2018). Este enfoque inclusivo muestra ejemplos magníficos de trabajo con niños y niñas de etnias vulnerables como la gitana, o con enfermedades graves como el cáncer, con mayores, con personas con discapacidad mental con los que se trabaja en clubes de lectura fácil, con inmigrantes, con personas reclusas, en centros psiquiátricos o en desempleo. 
En cambio, hay dos facetas en las que avanzar más: La primera es -aunque haya algunas prácticas dignas de mención ${ }^{1}-$, la relativa a la participación y colaboración ciudadana, que se podría aplicar más en el diseño y realización de proyectos y la formación de colecciones, la constitución de asociaciones de amigos y amigas de las bibliotecas, el voluntariado para la gestión o el apoyo en servicios, entre otros. $\mathrm{Y}$ la segunda es la reconfiguración de espacios, que potencien el uso social, la innovación y la colaboración (CARIDAD SEBASTIÁN et. al., 2018), pues las instalaciones están más pensadas para las colecciones o para usos como el estudio individual, y en los diez años de crisis no se ha podido abordar la reestructuración de los edificios y la renovación del mobiliario para dotar espacios más sociales.

\section{Objetivos y metodología}

En esta comunicación investigamos cómo se relacionan los discursos de los profesionales con la práctica de la función social que se lleva a cabo en el entorno municipal. Partíamos de varias preguntas de investigación: ¿Cómo consideran los profesionales que se materializa la función "social"? ¿Con qué limitaciones o dificultades cuentan? ¿Junto a qué otros profesionales deben ejercerla?

La metodología se ha basado en la aplicación de dos técnicas cualitativas: la entrevista y el grupo de discusión. Las entrevistas se realizaron a diez expertos, seleccionados por su alta experiencia profesional en entornos bibliotecarios, teniendo en cuenta la representatividad territorial (Andalucía, Aragón, Canarias, Castilla-La Mancha, Castilla y León, Cataluña, Comunidad Valenciana, Galicia, País Vasco y Región de Murcia) y contando en su haber profesional con tareas de representante de grupos de trabajo nacionales o internacionales, responsabilidad directiva en servicios o redes de bibliotecas, o haber desarrollado buenas prácticas de inclusión digital. Las entrevistas se realizaron en mayo de 2018, mediante un cuestionario virtual, estructurado en nueve cuestiones vinculadas con la función social: responsabilidad, requisitos, estrategias de desarrollo o rol a desempeñar por las bibliotecas públicas, entre otros. Las opiniones recogidas se categorizaron y analizaron, ofreciendo en este trabajo los discursos más relevantes, referidos, con número correlativo asignado, a cada experto (E1, E2 y así sucesivamente) para preservar su anonimato.

La opinión de los bibliotecarios municipales de la Región de Murcia se obtuvo mediante tres grupos de discusión, a los que asistieron de entre siete y nueve participantes,

\footnotetext{
${ }^{1}$ La Biblioteca Pública de Salamanca en la Casa de las Conchas aplicó una metodología de rediseño y prestación de servicios basada en la participación (Guerreiro, 2016), o el proceso del diseño de la nueva biblioteca en el barrio madrileño de San Fermín, realizada con la colaboración de la asociación de vecinos con los arquitectos y demás técnicos municipales (Ayuntamiento de Madrid, 2016).
} 
realizados en junio de 2017. Los grupos de discusión se clasificaron, en primer lugar, según el número de habitantes del municipio, considerando tres niveles (GD1: más de 50.000; GD2: entre 20-50.000 y GD3: menos de 20.000); además, dentro de cada grupo se consideró la situación geográfica y económica. Las sesiones tuvieron lugar en junio de 2018 en una sala de la Biblioteca Regional de Murcia, siguiendo en las tres un guion similar, estructurado en varios bloques de análisis vinculados con la función social (la responsabilidad de la biblioteca; la actitud y visión profesional; la colaboración con otros departamentos o entidades; la influencia de los colectivos usuarios; la biblioteca como espacio social y participativo; y la toma de decisiones sobre el desarrollo de esta función). Una vez transcritas las sesiones se procedió a categorizar las intervenciones de los participantes recogiéndose literalmente los discursos más relevantes e indicando entre paréntesis el número de participante, seguido del grupo de discusión (P1GD1, P2GD1, etcétera).

\section{Resultados}

Los resultados se han estructurado en dos partes; en primer lugar, se presenta la visión profesional de la función social: concepto existente de la misma, grado de asunción de la responsabilidad del servicio, actitudes y aptitudes percibidas, cambios profesionales que implica y condicionantes políticos que encuentran los bibliotecarios en su desarrollo. En segundo lugar, describiremos aspectos relacionados con la estructura y organización para el logro de la biblioteca social: requisitos (formación y competencias, recursos humanos y económicos), estrategias de ejecución colaborativa y en red, y adaptación a colectivos y usuarios destinatarios.

\section{a) Función social de la biblioteca}

a.1) Conceptualización

Los expertos consultados parten de distintas concepciones o maneras de entender a las bibliotecas como espacios de socialización y de participación social. Hay una gradación en este sentido. Mientras que para unos la biblioteca ya cumple una función social por el mero hecho de ser una institución pública de acceso abierto, gratuito y democrático, otros piensan que es indispensable que se implique más en el diseño y desarrollo de proyectos sociales, en fomentar la dimensión participativa y tener un papel más activo en materia de cohesión social y de lucha contra la exclusión.

Las bibliotecas tienen que convertirse en espacios inclusivos y neutrales para el intercambio cultural y social. Lugares de encuentro para que los ciudadanos puedan reunirse y relacionarse como agentes motores de desarrollo social de una comunidad (E1). 
Creo que las bibliotecas siempre han sido un espacio de socialización (más o menos rígido en función de cada época). La colección pierde protagonismo frente al espacio y a las propuestas de socialización. Sin embargo, es importante notar que espacio de socialización y participación ciudadana no son sinónimos. Hacer de las bibliotecas un espacio de socialización es algo natural para el colectivo de bibliotecari@s, aunque la apuesta por la participación ciudadana creo que puede no serlo tanto (miedos a que la gestión se nos escape, a la pérdida de la supuesta neutralidad que debemos ofrecer $y$ garantizar...) La biblioteca debe ser plural, inclusiva y no excluyente y apostar decididamente por la participación (E5).

Las bibliotecas deben postularse y conseguir que se nos vea como espacios de encuentro social y convivencia. Para ello tenemos que replantearnos nuestro papel de cara a la sociedad ya que hemos dejado de ser los espacios únicos de acceso a la información y al conocimiento. Por el contrario, debemos enfatizar el papel que desempeñan las bibliotecas como espacios de socialización y aprendizaje. Es importante que desarrollemos todo tipo de estrategias para fomentar la inclusión social y la participación ciudadana desde el espacio de la biblioteca pública (E10).

Sin duda y sin fisuras. Para muchos sectores de la población, y no solamente los excluidos, como hasta ahora se venía considerando, las bibliotecas son ese lugar donde cada día se encuentran, hablan y debaten con otros (E9).

Igualmente, los expertos mencionan los Objetivos de Desarrollo Sostenible como un nuevo referente para la biblioteca social.

Los Objetivos de Desarrollo Sostenible de Naciones Unidas para 2030 podrían guiar la actuación de las bibliotecas públicas también en este ámbito. En este sentido la IFLA ha desarrollado un gran trabajo explicando la relación y las actuaciones de las bibliotecas en todas y cada una de estas metas de sostenibilidad en un mundo cada vez más globalizado. (E10).

Las bibliotecas pueden ser instituciones fundamentales en la consecución de los Objetivos de Desarrollo Sostenible integrados en la Agenda 2030 de Naciones Unidas, a través de acciones de promoción de alfabetización universal incluyendo también las habilidades digitales (E4).

Por su parte, los técnicos bibliotecarios municipales manifestaron en los grupos de discusión su acuerdo con la función, afirmando que es la tarea más importante que tiene que asumir la biblioteca, ahora y, sobre todo, en el futuro.

Soy una defensora a ultranza de eso. La función social de la biblioteca es la más importante que tiene (P6GD1).

Yo creo que sí y es un ejercicio de responsabilidad que deje de ser ese patito feo y profundizar también esa función que hacemos y reclamarnos como un espacio donde suceden cosas cada día (P6GD3). 
a.2) Grado de responsabilidad

Los expertos manifiestan distintos grados de asunción de la responsabilidad que deben ejercer las bibliotecas públicas en el desarrollo de la función social en función de la concepción de la biblioteca de la que parten.

El hecho de ser espacios de socialización y participación no conlleva asumir ello como responsabilidad. Ser facilitador, promotor, incluso creador no necesariamente debe obligar a asumirlo como responsabilidad. Quizás es una cuestión de matices (E6).

Absolutamente. Las bibliotecas públicas deben liderar los procesos relacionados con la función social hacia la ciudadanía desde un punto de vista no asistencial sino de equidad, imparcialidad y legitimidad. $Y$ adaptando sus servicios para ser espacio de reunión, de intercambio y cohesión social (E3).

Claramente sí. Tenemos la responsabilidad de ser agentes dinamizadores de la sociedad y servicios públicos inclusivos. Tener un papel activo para que las personas salgan de la exclusión social, para que los ciudadanos tengan un acceso igualitario a la información, servicios donde se trabaje por la socialización de las personas...(E8).

Y los técnicos aun, asumiendo la responsabilidad, plantean la necesidad de coordinarse con otras unidades municipales como otros centros culturales, evitar el solapamiento de competencias y necesidad de cambiar la imagen de la biblioteca como lugar de estudio o silencio...

Hay centros culturales y sociales donde se está haciendo también eso. Entonces tenemos que ver hasta dónde debe hacerlo la biblioteca (P9GD1).

En el tema de las competencias, en ámbitos municipales, hay gente muy celosa de su parcela. Muchas veces te tropiezas con el vecino de al lado, que es tu compañero, que dice "no, para dar cultura ya tengo el auditorio" (P6GD3).

Sí, estoy de acuerdo, pero pienso que para traer a la sociedad a la biblioteca tiene que tener un espacio atractivo (P1GD3).

Solo excepcionalmente se afirma incapacidad para desarrollar la función de inclusión social por falta de ideas y de recursos:

No, porque no sabemos qué hacer (P7GD1).

a.3) Actitud y aptitud profesional como factor clave

Entre los expertos consultados hay gran coincidencia en destacar como elementos muy determinantes del desarrollo de la función social la actitud, la concienciación social y la formación del personal bibliotecario en este sentido, y, sobre todo, el primer elemento, la actitud, incluso por encima de los recursos económicos con los que cuentan. 
Básicamente actitud. Los conocimientos, habilidades y otras competencias son más fáciles de adquirir. La resistencia a cambios en el concepto de biblioteca es el más complejo de modificar (E6).

Es clave el factor humano, la naturaleza de l@s profesionales, nuestra capacidad de empatía, nuestro compromiso ético y moral y la red de complicidades que seamos capaces de tejer con la comunidad a la que servimos. Tener una actitud proactiva ante los diferentes colectivos sociales, conocer bien el tejido social para buscar colaboraciones. Ser extrovertidos, promover la participación y analizar otras experiencias puestas en práctica para adaptarlas a nuestra idiosincrasia (E5).

La clave está en la concienciación y formación del personal bibliotecario, en que esté convencido de que necesita trabajar colaborativamente con otras entidades sociales (E7).

Sin duda que el presupuesto y los recursos materiales van a tener que tenerse en cuenta para desarrollar tal función, pero más fundamental veo los profesionales encargados de llevarla a cabo y que, desde la planificación de la biblioteca, se tenga claro como una línea estratégica a desarrollar de forma inequívoca (E2).

También los técnicos bibliotecarios realzan el componente personal para poner en valor todo el potencial de la biblioteca, marcando un componente subjetivo en el ejercicio de la función: la predisposición, la carencia de prejuicios y la sensibilidad:

Estamos viendo que hay personas creativas: dejar de ser el patito feo y ese espacio que no cuenta poniéndolo en valor, sacando la biblioteca a la calle (P6GD3).

Aunque no nos parezca bien es la realidad: cada biblioteca tiene un estilo que le dan las personas que trabajan alli, no viene impuesto nada (P6GD1).

Formación sí, pero luego también está el cómo es la persona, los prejuicios... No sé hasta qué punto la formación afecta a la implicación (P9GD1).

a.4) Condicionantes políticos

Gran parte de los expertos coinciden en señalar la falta de políticas públicas para potenciar la dimensión social de la biblioteca, que además se acompaña de una falta clara de apoyo y compromiso político del que se deriva la falta de dotación presupuestaria y de recursos en general.

Considero que las administraciones públicas no tienen políticas para potenciar la biblioteca social, pero ni tan siquiera tienen una política bibliotecaria bien planificada y articulada (E7).

Las bibliotecas españolas están desarrollando claramente una función social, que con la crisis económica se ha puesto totalmente de manifiesto, pero falta una planificación real de como tienen que desarrollar esta función social y dotarlas de recursos (E8). 
Las competencias están perfectamente atribuidas. Pero otra cosa es que haya interés y preocupación por el tema. Siempre deberán ser las administraciones locales las más implicadas por la cercanía con los ciudadanos (E6).

En el caso de los técnicos municipales, en los grupos de discusión también mostraron sentirse a veces condicionados por los órganos de gobierno municipales cuando se trataba de abrir la biblioteca a la participación o permitir el uso de grupos o iniciativas de diverso signo político. Hubo discrepancia de opiniones sobre la toma de decisiones, sin estar siempre clara la neutralidad ideológica y la independencia profesional al desarrollar servicios. Se identifican algunas prohibiciones o represalias, en particular cuando se trata de actividades de tipo político o con colectivos específicos como inmigrantes.

El problema es que el de arriba te dice que no dejes (P4GD1).

Para según qué temas no te dan aprobación. Si ha habido una actividad con determinada orientación te han pegado un tirón de orejas (P2GD1).

Yo ahí me veo un poco más cobarde. Creo que lo consultaría (P6GD1).

El problema es que nosotros no tenemos autorización para acceder, tenemos que tramitarlo al ayuntamiento y que ellos lo decidan. Si son amas de casa no afecta. Antes de tener las Salas 24 horas se dejaba la llave a los estudiantes. Yo creo que sí debería acoger estas actividades, pero con medidas de control, que haya un responsable o un papel que se firme... (P5GD3).

Yo he pasado de un concejal muy pendiente porque la biblioteca estaba en la cresta de la ola, a que la concejala ni me mire. Y no le interesa nada más que no haya comentarios malos de la biblioteca en las redes sociales (P7GD3).

A mí no me dice el concejal "no dejes entrar" o "si". Nosotros informamos al concejal de qué queremos hacer, porque en nuestro caso hay libertad total por el desconocimiento que hay. Igual que hay cierta dejadez, que es mala, tenemos cierta libertad, lo que es bueno (P6GD1).

Creo que todo depende no de los políticos sino de las personas, a lo largo de los años han pasado muchos, y hay buenos, malos y regulares. No depende del partido sino de la persona. $Y$ de cómo vendamos nosotros la biblioteca, porque cuando han pasado más les he enseñado lo que hacemos (P5GD3).

a.5) Repercusiones sobre la visión profesional

Una cuestión que se deriva es cómo encajar la nueva función, que supone nuevas tareas, con las muchas que ya vienen realizando los profesionales. Independientemente de la necesaria incorporación de más personal a las bibliotecas mal dotadas, conduce a los

bibliotecarios a replantearse su orden de prioridades, o en ocasiones a una sensación de estrés laboral al acumularse nuevos compromisos o retos, a pesar del voluntarismo.

Tienes que hacer de psicólogo, de cuentacuentos, de informático. Es que el nexo que hay entre el usuario y el bibliotecario en una biblioteca pequeña es primordial y lo es todo. Hay más humanización y te implicas en problemas del usuario porque llegan a contarte algo de su vida y pedir consejo (P3GD3).

Yo llevo más de 25 años de servicio y estoy cansada. He trabajado mucho y no siempre se ha reconocido lo que he hecho. He visto de todo, he tenido muchos 
concejales distintos, y a veces no han apoyado la biblioteca, no la entendían. Estos últimos años han sido muy duros: poco presupuesto, pocos ordenadores y poco personal de apoyo. A veces he estado sola y he intentado cubrir unos mínimos. No sé...(P1GD2).

Un inconveniente cuando abres la biblioteca a distintos usos es que debes devolver a la biblioteca a su estado supuestamente natural. Cuando hemos montado ludotecas con chavales luego tienes cierta responsabilidad con el orden alfabético, los préstamos, el carnet que tengo retrasado... Las funciones "puramente bibliotecarias" se ven retrasadas o entorpecidas; es llevadero, pero es verdad que a mí me cuesta devolver a la biblioteca más o menos a su estado continuo" (P6GD3).

Me da satisfacción: cuando ayudas a alguien que del ayuntamiento han mandado a la biblioteca a que le hagamos la Renta o los papeles del paro. Y si no hacemos eso se nos va la gente (P1GD3).

En mi caso lo veo positivo, porque la función de la biblioteca es enriquecer el entorno. Si que a veces te toca remar y correr, y piensas: "¿qué necesidad tengo yo?'. Pero me merece la pena el sacrificio porque veo resultados en mi pueblo y veo que se va por el buen camino (P4GD3).

Hay una predisposición del usuario a llegar y pedir una cosa, y aunque no corresponda a nuestras funciones, tú estás dispuesto a hacérsela (P7GD3).

Yo ya he explicado la orfandad en la que nos sentimos muchas veces (P6GD2).

\section{b) Aspectos de estructura y organización para la función social}

b.1) Necesidades y requisitos

La respuesta de los expertos sobre los requisitos de estructura y organización para el desarrollo de la función social por parte de las bibliotecas es muy variada. Aluden a necesidad de formación, de competencias para el diseño de proyectos y a recursos humanos y económicos.

Disposición, formación y recursos (E9).

Es clave poder contar con espacios físicos flexibles, amables, sugerentes $e$ imaginativos que permitan diferentes usos y favorezcan el acercamiento de grupos y ciudadanos, sobre todo los tradicionalmente ajenos y alejados (E5).

Se necesitan recursos, materiales y personales, buenos espacios, pero sobre todo planificar y desarrollar buenos programas que contribuyan y fomenten que las bibliotecas ayuden a corregir los desequilibrios sociales (E8).

En principio, los profesionales y su forma de actuar es un elemento clave. Los equipos de profesionales deberían ser multidisciplinares y con habilidades específicas para gestionar situaciones producto de una biblioteca con una función social. La flexibilidad, adaptabilidad y el conocimiento del entorno son otro factor clave: tipología de usuarios, procedencia, nivel de estudios, y condicionantes culturales. Finalmente, el presupuesto es un elemento clave, ya que al planificar el servicio bibliotecario, deberá tenerse en cuenta la inclusión de profesionales de la educación social al equipo de técnicos (E3). 
Solo uno de los expertos consultados da una respuesta integral que abarque los tres tipos de competencias formativas (competenciales, procedimentales y actitudinales).

A nivel de conocimientos es necesario conocer las políticas de cohesión y participación social, fomentar la interrelación y el respeto por la diversidad sociocultural, saber planificar servicios accesibles y actividades inclusivas $y$ trabajar en entornos digitales. A nivel de habilidades es muy importante la capacidad para resolver problemas, para trabajar en equipo; para adecuarse a las necesidades de los usuarios y para crear redes de colaboración y aprendizaje. $Y$, por último, a nivel de valores es fundamentar la defensa de una serie de valores éticos y democráticos como son la igualdad, el respeto, la solidaridad, la tolerancia y la justicia social (E4).

Insisten también sobre la falta de recursos económicos.

Sin duda el presupuesto y los recursos materiales, de equipamientos, infraestructuras y personal van a tener que tenerse en cuenta para desarrollar la función, pero más fundamental es que desde la planificación de la biblioteca se tenga claro como una línea estratégica a desarrollar de forma inequívoca (E2).

Sin lugar a dudas los recursos (espacios, presupuestos, tecnologías, RRHH) son necesarios para hacer proyectos sociales, pero no son los únicos ni los primordiales. Tenemos claros ejemplos en Centro y Sudamérica de exitosos proyectos sociales hechos con muy pocos recursos (E7).

Los técnicos bibliotecarios manifestaron en los grupos de discusión la necesidad de formación en competencias para atender a colectivos específicos de todo el personal bibliotecario, desde el conserje al director, que esté apoyada y gestionada desde la Red y la Biblioteca Regional, y que vaya acompañada de dotación presupuestaria, de reglamentación normativa y de un reconocimiento de sus tareas para que sea efectiva.

Necesitamos formación para el personal que atiende al público (P6GD1).

A veces es que no sabemos cómo; necesitamos apoyo. Eso lo podría hacer la Biblioteca Regional, marcando una línea (P7GD1).

Se debería cambiar la relación de tareas en la Relación de Puestos de Trabajo. Porque, igual que estamos viendo que las funciones en una biblioteca están cambiando, también se deben actualizar todas las funciones que tienen los técnicos, auxiliares y ordenanzas. (P2GD1).

b.2) Estrategias de ejecución

Hay unanimidad entre los expertos sobre la necesaria implicación de otros agentes e instituciones en el desarrollo de esta labor.

La biblioteca sola está muerta, así de triste y así de sencillo. Tenemos que trabajar con los profesionales que nos rodean: colegios, profesores, sociólogos, asistentes sociales, responsables de las instituciones sociales... Aquella máxima 
de "aquí estamos, el que quiera que venga" nos aboca a una larga agonía y una muerte segura... (E9).

Si una lección podemos sacar de este mundo conectado en que vivimos es la constatación de que ninguna institución, entidad u organismo, por muy grande y poderosa que sea, podrá por sí misma, conseguir el objetivo. Si queremos tener éxito debemos tener muy presente, que solos, no podemos (E5).

Es imprescindible el compromiso de la administración pública de la que depende la biblioteca y es necesaria la colaboración, implicación y cooperación de agentes/entidades sociales, educativos, de mediación y de aprendizaje (E4).

El tejido y el entramado social se convierten en necesarios aliados. Nosotros los bibliotecarios, sabemos de lo que sabemos... Por ello, debemos aliarnos y dejarnos guiar en determinados aspectos para poder desarrollar el trabajo de forma completa (E9).

El/la bibliotecario/a no puede llegar a ser multidisciplinar, pero sí puede redirigir su gestión para que la biblioteca se convierta en un espacio flexible, multifuncional y orientado a la colaboración, lo más permanente posible, con diferentes agentes de desarrollo social. Servicios sociales, servicios sanitarios, centros educativos. (E1).

Una parte importante de los expertos considera que debe impulsarse un plan estratégico que integre la cooperación entre las distintas administraciones para que se asuma de manera real la función social de las bibliotecas. Tanto desde el ámbito Estatal, como el autonómico, permitiendo una mayor adaptación a la realidad social y la local por su cercanía a la ciudadanía.

Creo necesario establecer planes regionales estratégicos y de comunicación que den unidad de actuación y que potencien y den visibilidad a la imagen de la biblioteca desde su dimensión social (E1).

Es indispensable un Plan estratégico nacional con acuerdos de colaboración y financiación con la administración autonómica. Planes autonómicos de acuerdo a necesidades socio-económicas y culturales de territorio. Estrecha coordinación/colaboración con administración local para diseño de planes que den coherencia y unidad a la actividad de la biblioteca (E1).

Son las administraciones locales, puesto que son las más cercanas a la ciudadanía, las que tienen que tener las competencias directas en todo lo que tiene que ver con la ejecución de los proyectos de bibliotecas y las otras dos Administraciones deben de ejercer funciones de planificación y coordinación e impulso de proyectos innovadores en las bibliotecas de su territorio (E7).

Los bibliotecarios de los municipios son los que ponen en práctica buenas iniciativas de cooperación, apoyadas en la colaboración con distintas instituciones -sobre todo organizaciones del Tercer Sector como CEPAIM, Cáritas, Traperos de Emaús, ASIDO, APANDI...para trabajar con distintos colectivos (personas gitanas, desempleadas, con discapacidad, menores, o mayores) en proyectos de donación de alimentos, clubes de lectura fácil... 
Tenemos un montón de programas con cada sector de la población (P2GD2).

Hemos estado colaborando con el centro penitenciario, se ha formado allí un club de lectura en la prisión (P3GD1).

Es interesante que colaboremos con otras concejalías porque atraen público que normalmente no va a la biblioteca. Es una forma de darte a conocer (P7GD1).

En nuestro caso, ya estamos en contacto la Concejalía del Mayor, la de Juventud y la de Nuevas Tecnologías. Cuando hemos hecho cursos con mayores, en colaboración con la Concejalía del Mayor, ellos aportaban un técnico, y nosotros explicábamos cómo se usaba "Ebiblio" (P6GD1).

El conocimiento y dar más visibilidad a las buenas prácticas es otra estrategia para potenciar esta función, y por ello destacan la labor de difusión de los proyectos sociales y solidarios desarrollados desde Fundación Biblioteca Social:

Necesitamos conocer qué se está haciendo en otras bibliotecas; no hace falta ser originales ni pioneros. Todo está inventado: es interesante tomar de otros lo que está bien hecho y simplemente adaptarlo a nuestros intereses (E2).

Hay buenas prácticas y están recogidas en la web de la Fundación Biblioteca Social (E7).

Otras de las estrategias expuestas tienen que ver con los retos que actualmente enfrentan las bibliotecas para desarrollar adecuadamente su función social. La mayor parte de los retos apuntados por los expertos tienen que ver con conseguir un cambio de imagen de las bibliotecas que visibilice la utilidad social de su labor, y en el que tiene gran importancia la transformación de las instalaciones físicas:

Entre los retos de cara al futuro se encuentra el cambiar la imagen social que se tiene de la biblioteca y conseguir que se defina más por los servicios que se ofrecen a los usuarios que por lo que contiene. $Y$ en este cambio de papel tendrá un gran impacto el espacio físico y la forma de cómo la comunidad la hace suya e interactúa. Las bibliotecas como espacios de creación que pueden aumentar la participación ciudadana diseñando espacios para experimentar con las nuevas tecnologías, en las que relacionarse y compartir el trabajo en común. Es el llamado "movimiento maker" y los nuevos makerspaces en las bibliotecas, centros de recursos tecnológicos que se ponen a disposición de la comunidad (E10).

El mayor reto es lograr un cambio de imagen. Los usuarios aún identifican la biblioteca con un espacio para estudiar y no para la convivencia y el intercambio de experiencias (E3).

Propiciar la participación de la población con un enfoque global e integral como prevención de situaciones de exclusión social y diseñar propuestas que den visibilidad al servicio y sirva para la democratización del mismo (E1).

Y manifiestan el papel dinamizador de los Objetivos de Desarrollo Sostenible: 
Integración del desarrollo de las Bibliotecas Públicas, haciendo especial hincapié en su rol social, en la estrategia política como que pueden ser fundamentales en la consecución de los Objetivos de Desarrollo Sostenible a través de diversas acciones (E4).

b.3) Adaptación a colectivos destinatarios

La atención a la diversidad de usuarios es percibida como una riqueza, y su conocimiento necesario para el buen servicio, que parecen orientar hacia los más vulnerables.

Entre los factores que más inciden en el potencial para desarrollar la función social de las bibliotecas podemos considerar la población a la que sirve y la tipología de usuarios que la frecuentan (E10).

Uno de los mayores potenciales con los que cuentan las bibliotecas es el amplio abanico y perfil de usuarios reales y potenciales (E1).

La flexibilidad, adaptabilidad y el conocimiento del entorno son otro factor clave: tipología de usuarios, procedencia, nivel de estudios, condicionantes culturales, etcétera. (E3).

Los grupos socialmente más desfavorecidos deben ser los primeros beneficiarios. Personas de más edad, para muchos de los cuales la tecnología puede llegar a convertirse en un muro infranqueable, personas con escasa formación académica, desempleados o inmigrantes. (E10).

Los técnicos de las bibliotecas municipales relatan ocasionalmente situaciones problemáticas con algunos usuarios, que llevan a un trato diferenciado que podría convertirse en conflictivo:

Pero claro, yo estoy sentada y tengo al lado un señor que huele mal. ¿Cómo gestionas eso? ¿Cómo digo a este hombre que vive en la calle y no tiene la posibilidad de ducharse que huele mal? No puedes cerrar la puerta (P5GD1).

Yo sí que he tenido que llamar a la policía para levantar a alguien de su sitio que no me hacía caso (P6GD3).

\section{Conclusiones y propuestas de intervención}

\subsection{Alto compromiso con la función social, identificando ésta de modo prevalente con la} atención a sectores más vulnerables

Hay un alto compromiso con la función social por parte de los profesionales de las bibliotecas, partiendo de una de sus dimensiones, el carácter abierto a todas las personas (enfoque general), pero muy enfocada también a la dimensión que hemos llamado compensatoria, orientada a los colectivos vulnerables social o digitalmente, sobre todo mayores y desfavorecidos por condición de procedencia inmigrante o económica. La dimensión participativa y de colaboración se ve condicionada por una sensación de control político y la inadecuación de las instalaciones, y respecto a la dimensión orientada a la 
sostenibilidad, se está empezando a introducir, de modo incipiente, el papel estratégico de vincular las bibliotecas con los Objetivos de Desarrollo Sostenible, y contribuir a ellos en la medida de sus posibilidades, como una faceta de la función social.

5.2. Existencia de condicionantes para el desarrollo de la función social, aunque siendo determinante la actitud profesional

El desarrollo de esta función, que intentan llevar a cabo de acuerdo con las demandas y necesidades de su contexto local, se ve condicionado por aspectos vinculados con los recursos (humanos y materiales) con los que se cuenta, la formación, el tipo de usuarios y su diversidad (cultural, etaria). La persistencia de falta de recursos, la adición de nuevas tareas y, en ocasiones, la precariedad laboral son factores que explican cierta sensación de agobio o estrés laboral.

En esta situación se hace muy determinante la actitud del personal, su nivel de concienciación y convencimiento del carácter prioritario de estas funciones, para incluso resituarlas dentro del conjunto de sus tareas y así darles más cabida. Por ello, a pesar de predisposición puede estar habiendo un desarrollo desigual de estas funciones.

Respecto al factor "político", observamos críticamente que hay un insuficiente nivel de autonomía en la toma de decisiones por una tendencia al control municipal, que puede llevar a una inhibición en el fomento de la participación comunitaria en la biblioteca o a la prevención respecto a determinados grupos políticos o determinadas nacionalidades de población extranjera.

\subsection{Necesidad de liderazgo, coordinación interna y alianzas con agentes externos}

Se realizan muchos esfuerzos creativos y se busca la colaboración con otros departamentos o agentes sociales (públicos o privados) e incluso con los usuarios, pero creemos que deben ser acompañados por un apoyo que reivindican los bibliotecarios: que la Biblioteca Regional ejerza un liderazgo y su responsabilidad de coordinación. Lo idóneo sería el diseño de un plan estratégico regional que articulara las actuaciones y estabilizara estas funciones en las infraestructuras bibliotecarias de los municipios, pero al menos se debe:

- Reactivar los planes de formación profesional continua, incluyendo una conceptualización de la función social y sus desarrollos prácticos. Una reflexión teórica sobre lo que significa ser una biblioteca social y educadora en el contexto actual, luego conducente a una ampliación de las prácticas profesionales

- Mejorar el reconocimiento profesional y normativo, materializado en las Relaciones de Puestos de Trabajo y en la actualización de las Normas para los servicios bibliotecarios de la Región. 


\subsection{Fomento institucional de la función social y actuaciones para su impulso}

Para potenciar la función social proponemos cuatro medidas cuya factibilidad puede depender de la cofinanciación autonómica o central a través de una convocatoria pública:

- Un plan de remodelación de infraestructuras de las bibliotecas públicas para reformar espacios con el fin de hacerlos más flexibles y abiertos. En coherencia con un enfoque más social, de encuentro, de colaboración y de creación, y con el contexto digital, las bibliotecas deben hacer un nuevo aprovechamiento y distribución de sus espacios, quizás retirando colecciones o puestos de estudio individual para permitir un mejor y más diverso aprovechamiento de las instalaciones. Para facilitar esta transformación se debería elaborar unas Pautas para espacios y equipamiento de bibliotecas con enfoque social, realizada por un grupo de bibliotecarios y arquitectos seleccionados por su experiencia en el diseño de estos nuevos espacios bibliotecarios

- Implantar en las bibliotecas públicas un punto de información y formación sobre transparencia, participación y e-ciudadanía, para orientar sobre cómo hacer peticiones, reclamaciones u obtener información a través de los Portales de Transparencia, y contribuir a hacer efectivo el derecho a saber. El servicio pretendería -previa una acción formativa destinada al propio personal- informar, canalizar y enseñar sobre cómo obtener la información, integrarse en procesos de presupuestos participativos o similares, y creemos que potenciaría la imagen de la biblioteca como un espacio de confianza y de los profesionales como defensores del derecho a la información desde la neutralidad, el respeto a la diversidad y la confidencialidad establecidos en el Código de ética para bibliotecarios y profesionales de la información de España (2013).

- Incorporar mobiliario y equipamiento que facilite el uso por parte de personas en exclusión residencial: las bibliotecas deben instalar taquillas de tamaño amplio para la custodia de objetos personales de quienes llegan a la biblioteca con sus pertenencias, e instalar una ducha en cada uno de sus cuartos de baño.

- Mejorar la disponibilidad de tecnologías digitales para personas vulnerables: En lo posible es recomendable hacer una nueva distribución de los ordenadores de uso público ubicándolos en distintos espacios en lugar de en una sala cerrada, para prevenir la guetificación que puede estar aconteciendo en estos lugares. E implantar el préstamo de larga duración de portátiles para personas que no puedan disponer de esta herramienta, en periodos mensuales renovables en función de la demanda.

\subsection{Hacia una biblioteca más social}

Los bibliotecarios españoles son conscientes de la importancia de su rol social, sobre todo en el sentido "inclusivo", y así lo expresaron en el Estudio promovido por FESABID (MERLO-VEGA, GÓMEZ-HERNÁNDEZ, HERNÁNDEZ-SÁNCHEZ, 2011), en el que "la convicción de que las bibliotecas pueden contribuir en el acceso igualitario y la reducción de la exclusión de la sociedad del conocimiento, contribuyendo a la integración, inclusión y desarrollo social y cultural" se consideró una oportunidad para los profesionales de la información. Y la reciente 
Visión Global consensuada por IFLA (2018) a través de la respuesta masiva a una consulta mundial afirma que "debemos entender mejor las necesidades de la comunidad y diseñar servicios que tengan impacto. La ampliación del alcance de las bibliotecas ayudará a crear vínculos con los socios locales, incorporar nuevos sectores de nuestras comunidades o sectores con servicios deficientes, y tener un impacto mensurable en la vida de las personas".

Se actúa, pero hay mucho que avanzar todavía: el reto profesional es materializar estas convicciones haciendo prioritaria la función social y articular los medios materiales, humanos y conceptuales para hacerla posible.

\section{Referencias}

AYUNTAMIENTO DE MADRID. La biblioteca de San Fermín, un proyecto fruto de la colaboración entre vecinos y el Ayuntamiento. Ayuntamiento de Madrid. 2016. Retrieved: October 16, 2018. From <https://bit.ly/2PQflBU>

CARIDAD-SEBASTIÁN, M.; GARCÍA-LÓPEZ, F.; MARTÍNEZ-CARDAMA, S.; MORALES-GARCÍA, A. M. Bibliotecas y empoderamiento: servicios innovadores en un entorno de crisis. Revista Española de Documentación Científica, v. 41, n. 2, p. 1-14, 2018 <https://doi.org/10.3989/redc.2018.2.1486>

CASTILLO-FERNÁNDEZ, J. Biblioteca punto de empleo. In: CASTILLO-FERNÁNDEZ, J.; GÓMEZHERNÁNDEZ, J. A.; QUÍLEZ-SIMÓN, P. (Eds.). La biblioteca pública frente a la recesión, acción social y educativa. Murcia: Tres Fronteras. 2010 . p. 173-188.

CÓDIGO DE ÉTICA PARA BIBLIOTECARIOS Y PROFESIONALES DE LA INFORMACIÓN DE ESPAÑA. Madrid: Federación Española de Sociedades de Archivística, Biblioteconomía, Documentación y Museística, 2013. <http://www.fesabid.org/sites/default/files/repositorio/codigo-eticoesp.pdf>

CONSEJO DE COOPERACIÓN BIBLIOTECARIA. GRUPO DE TRABAJO FUNCION SOCIAL DE LA BIBLIOTECA. Decálogo Biblioteca social. España, 2017. Retrieved from: <http://www.ccbiblio.es/wp-content/uploads/Decálogo-Biblioteca-social.pdf>

D'ALÒS-MONER, A.; BAILAC, A.; HERNÁNDEZ-SÁNCHEZ, H. Dimensión social de las bibliotecas públicas. Anuario ThinkEPI, v. 9, p. 66-71. 2015. <https://doi.org/10.3145/thinkepi.2015.12>

DOMíNGUEZ-SANJURJO, R. El cambio invisible. Biblioteca y sociedad. Boletín de la Asociación Andaluza de Bibliotecarios, n. 113, p. 21-28, 2017. Retrieved from: <https://www.aab.es/publicaciones/boletín-aab/boletín-113/>

ELMBORG, J. K. Libraries as the Spaces Between Us: Recognizing and Valuing the Third Space. Reference \& User Services Quarterly, v. 50, n. 4, p. 338-350, 2011. $<$ https://doi.org/10.2307/20865425>

FUNDACIÓN BIBLIOTECA SOCIAL. Mapa de proyectos. Convocatoria 2017. 2018. <https://fundacionbibliotecasocial.org/es/categories-map/convocatoria-2017-es/>

GÓMEZ-HERNÁNDEZ, J.-A.; HERNÁNDEZ-PEDREÑO, M.; ROMERO-SÁNCHEZ, E. Social and digital empowerment of vulnerable library users of the Murcia Regional Library, Spain. EI Profesional de La Información, v. 26, n. 1, p. 20-33, 2017. <https://recyt.fecyt.es/index.php/EPI/article/view/epi.2017.ene.03/33295> 
GUERREIRO, J. Espacio compartido: Modelo de relación biblioteca - comunidad basado en la participación ciudadana y la teoría de los stakeholders. 2016. <http://hdl.handle.net/10366/133019>

IFLA. Lyon Declaration on access to information and development. 2014. $<$ htttp://www.lyondeclaration.org/>

IFLA. Access and oportunities for all. How libraries contribute to the United Nations 2030 Agenda. The Hague: IFLA. 2016 Retrieved from: $<$ https://www.ifla.org/files/assets/hq/topics/libraries-development/documents/access-andopportunity-for-all.pdf>

IFLA. Guidelines for Library Services to People Experiencing Homelessness. The Hague, Netherland. 2017. Retrieved from: <https://www.ifla.org/files/assets/lsn/publications/iflaguidelines-for-library-services-to-people-experiencing-homelessness.pdf>

IFLA. Global Vision: Top 10 Highlights and Opportunities. 2018. <https://www.ifla.org/files/assets/GVMultimedia/publications/gv-report-summary_2.pdf>

MERLO-VEGA, J.-A.; CHU, C.-M. Out of Necessity Comes Unbridled Imagination for Survival: Contributive Justice in Spanish Libraries during Economic Crisis. Library Trends, v. 64, n. 2, p. 299-328, 2015. <https://doi.org/10.1353/lib.2015.0051>

MERLO-VEGA, J.-A., GÓMEZ-HERNÁNDEZ, J.-A.; HERNÁNDEZ-SÁNCHEZ, H. Estudio FESABID sobre los profesionales de la información: prospectiva de una profesión en constante evolución. Madrid: Federación Española de Sociedades de Archivística, Biblioteconomía, Documentación y Museística, FESABID.2011. <https://doi.org/10.13140/RG.2.1.2489.6080>

PÉREZ-SALMERÓN, G. Acceptance Speech. In: IFLA WORLD LIBRARY AND INFORMATION CONGRESS. Libraries. Solidarity. Society. Wrocław (Poland)., 2017. Retrieved from: <https://www.ifla.org/node/11791>

TOGORES, R. El valor de las bibliotecas públicas en la sociedad: El caso de la red de bibliotecas municipales. Barcelona: Diputació de Barcelona, 2014. Retrieved from: <http://www.diba.cat/documents/16060163/22275360/valor_social_bibliotequesXBM_cast.p df/02926665-2d7e-4044-88c2-9634abed0c89> 